\title{
Formulation of the Road Supply Index Based on the Land Use Patterns and Street Functions for Major Residential Areas
}

\author{
Jaisung Choi*, Sangyoup Kim**, Youngsoo Jang***, and Myungsoo Kang****
}

\section{Erratum to:KSCE Journal of Civil Engineering (2008) 12(5):329-338 \\ DOI 10.1007/s12205-008-0329-0}

The original version of this article unfortunately contained a mistake. In Table 16, Name of Site column:

Total Land Area() (A) $\rightarrow$ Total Land Area $\left(\mathrm{m}^{2}\right)(\mathrm{A})$

Load Area ()$\rightarrow$ Load Area $\left(\mathrm{m}^{2}\right)$

*Member, Prof., Dept. of Transportation Engineering, University of Seoul, Seoul 130-743, Korea (E-mail: traffic@uos.ac.kr)

**Ph.D. Student, Dept. of Transportation Engineering, University of Seoul, Seoul 130-743, Korea (Corresponding Author, E-mail: road@uos.ac.kr)

***Team Director, Management Information Office, Korea Land Corporation, Kyunggido 463-755, Korea (E-mail: ysjang60@hanmail.net)

****Ph.D., Human-Environment, Land\&Urban Institute, Korea Land Corporation, Kyunggido 463-755, Korea (E-mail: juniatoki@nate.com) 